R ff $\quad$ ff

\title{
Academic management by processes: An efficient proposal for Politecnica Salesiana University
}

Fernando Pesantez Aviles ${ }^{a}$ *

Andrea Plaza Corderob

Suggested Citation

$\mathrm{R}$

R

) . fff

ff $\mathrm{R}$

$\mathrm{R}$

$\mathrm{R}$

fff $R$

$\mathrm{ffR} \quad \mathrm{ff}$

$\mathrm{ff}$

$\mathrm{R}$

Abstract

ff

ff fff

$\mathrm{R}$ ff

$\mathrm{R}$ fff

ff

R

ff $\mathrm{R} \quad \mathrm{fff}$
R

ff

$\begin{array}{lll}\mathrm{ff} & & \\ \mathrm{ff} & & \\ \mathrm{ff} & & \\ & \mathrm{ff} & \end{array}$

fff 
1. Introduction

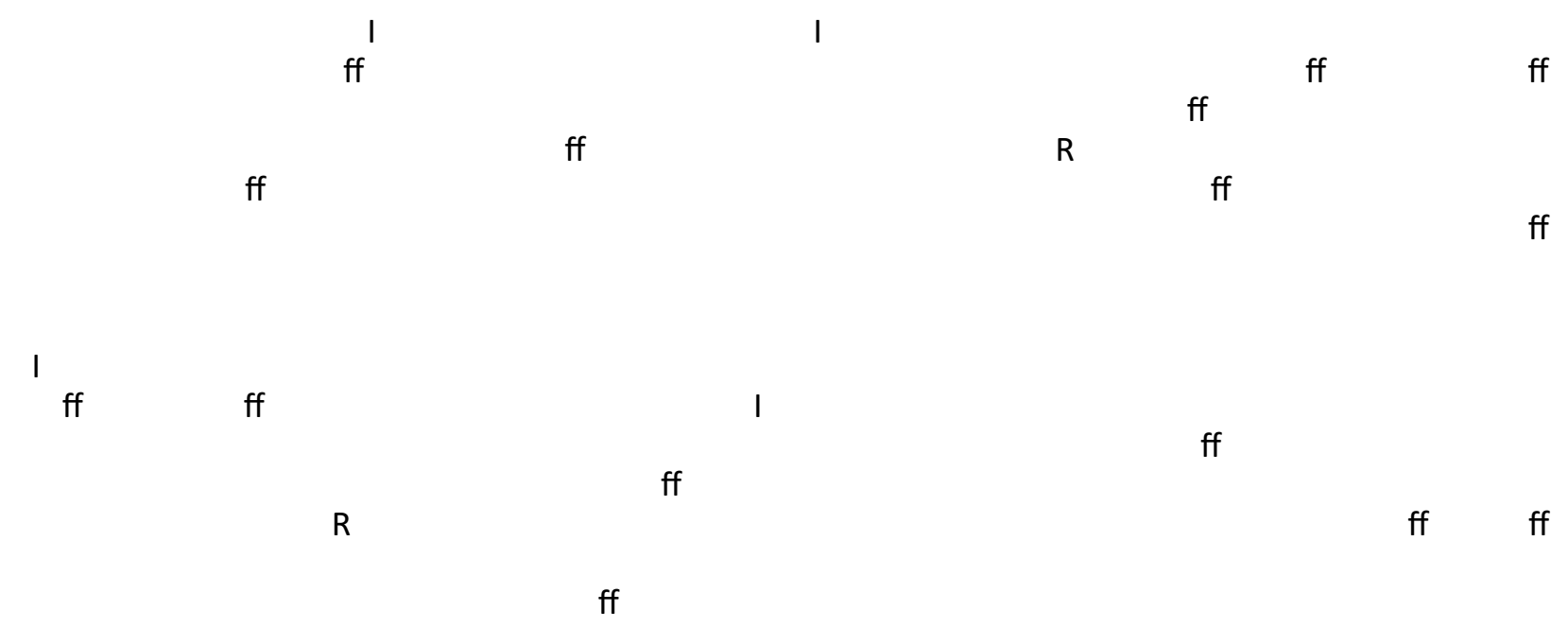

Table 1. Reforms of educational regulations in Ecuador

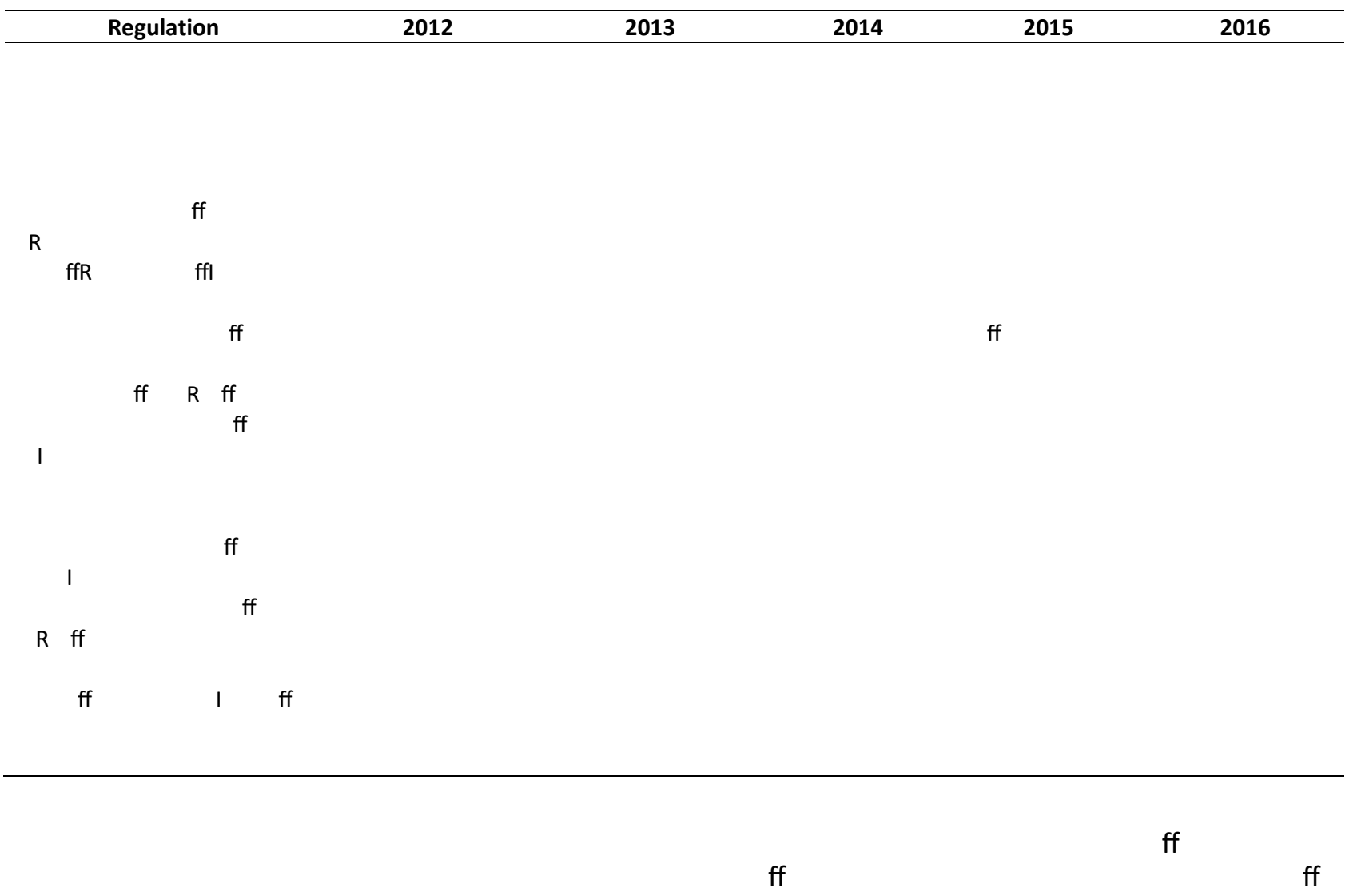


R

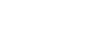

ff

ff R

2. Method

R $\quad f$

ff

- $\mathrm{ff} \mathrm{ff}$

R

ff ff

ff

fff

ff

ff

ff

ff $\mathrm{ff}$

ff

R

ff

ff

ff fff

ff $\mathrm{R}$

ff

ff

ff

ff

ff

ff

ff

ff

fff $\quad$ ff

ff

ff

ff

ff

ff

ff 
$\mathrm{ff}$

ff ff ff

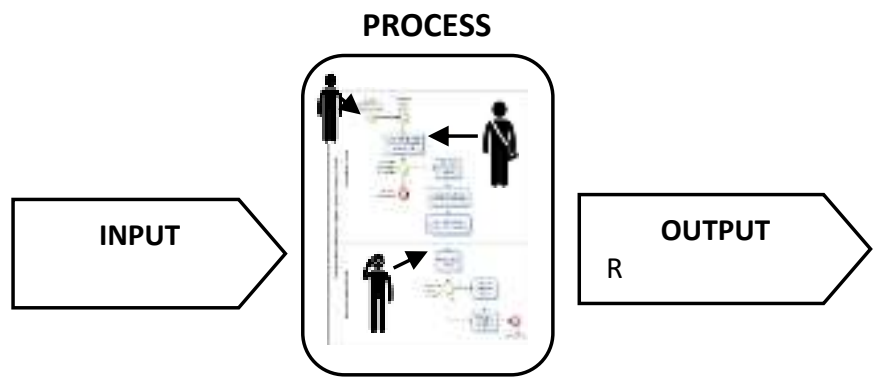

Figure 1. Elements of the Processes

ff

R

ff I

$\mathrm{ff}$

- 1

- $\quad \mathrm{ff}$

$\bullet \quad \mathrm{ff}$ ff

ff ff

3. Material and Method

$\mathrm{R}$

$\mathrm{ff}$

R

ff $\mathrm{ff}$

ff ff

ff

ff

ff

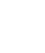


R

R ).

ff

$f$

$\begin{array}{llllllll} & & \mathrm{ff} & \mathrm{ff} & & & \\ \mathrm{ffl} & & & \mathrm{ff} & & & \\ \mathrm{ff} & & & & \mathrm{ff} & & \mathrm{ff}\end{array}$

$\mathrm{R}$

ff

ff

Stage 0 (Education and Training):

ff

$\mathrm{ff}$

ff

ff

Stage 1 (Identification of the academic processes):

ff

ff

ff

ff

ff

Table 2. Identification of academic processes

\begin{tabular}{|c|c|c|c|}
\hline Macro Process & Process & Codification & Priority \\
\hline & & & I \\
\hline & & $\mathrm{R}$ & \\
\hline & & R & $\begin{array}{l}1 \\
1 \\
1 \\
1 \\
1\end{array}$ \\
\hline $\mathrm{ff}$ & $\begin{array}{c}38 \\
\text { process }\end{array}$ & $\begin{array}{l}\mathrm{R} \\
\mathrm{R}\end{array}$ & 1 \\
\hline
\end{tabular}

Stage 2 (Establishment of formats/templates): ff $\mathrm{ff}$ $\mathrm{ff}$ 
ff

- 1

- $\quad$ ff $R$

ff

ff $\quad$ R ff $\mathrm{ff}$

ff ff

ff

Stage 3 (Assign people in charge):

$\mathrm{ff}$

$\mathrm{ff} \mathrm{ff}$

ff

$\mathrm{ff}$

ff

$\mathrm{ff}$

ff

Stage 4 (Creation of the process):

ff

ff

- Review of information/regulations: ff

ff ff

- Approval of the draft:

ff

- Presentation to expert users:

ff

fff $\quad \mathrm{ff}$

ff

ff

ff $\quad \mathrm{ff}$

- Generation of the preliminary version: ff

- Diagram: 


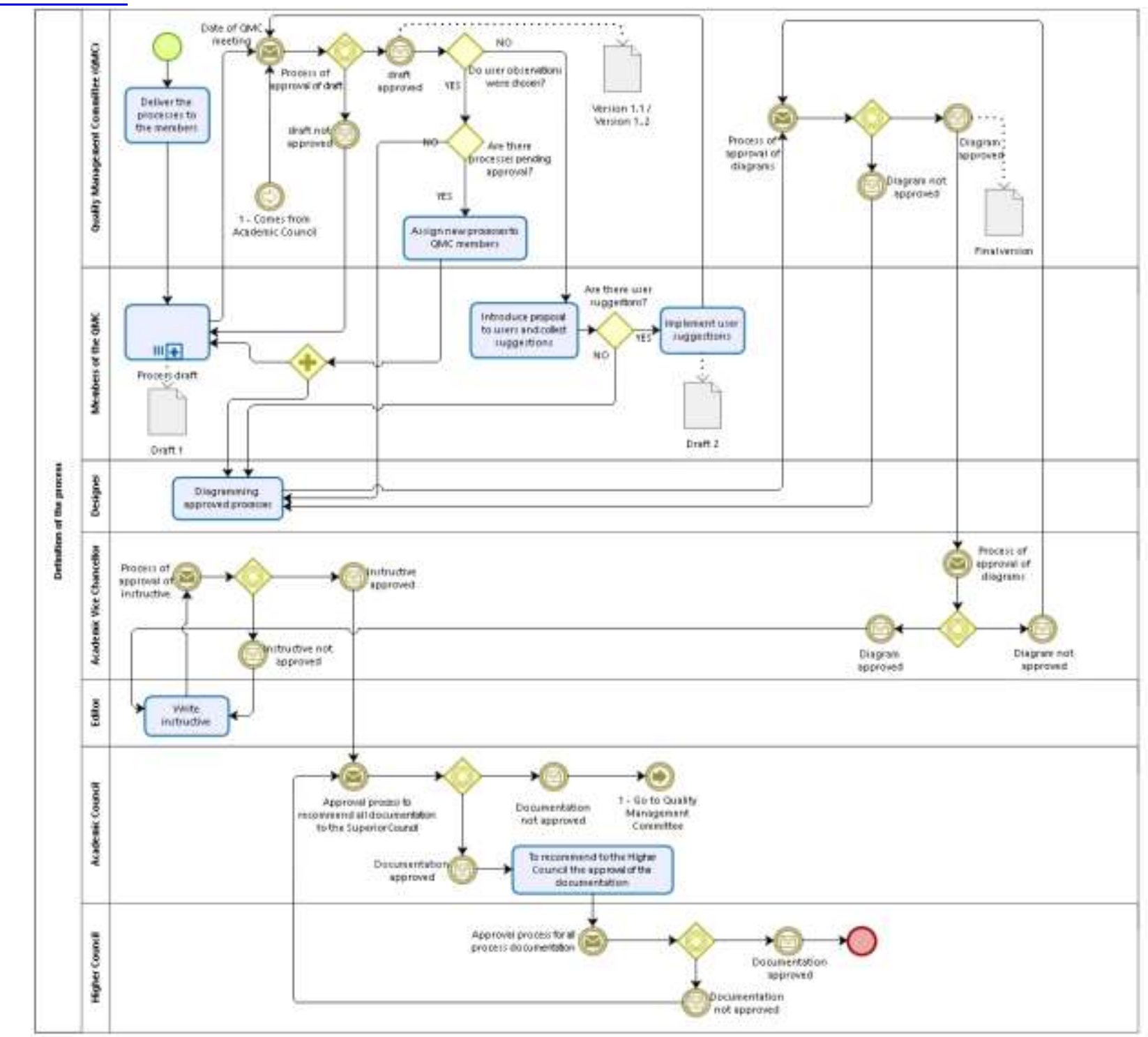

Figure 2. Process Creation

\section{Stage 5 (Review and approval):}

$$
\text { ff }
$$

$\mathrm{ff}$

f

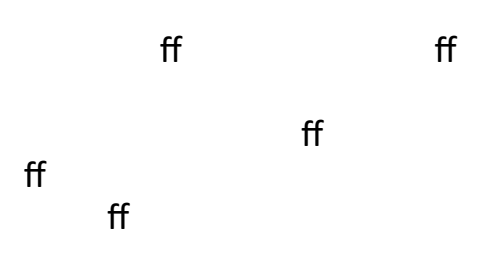


Figure 3. Analysis and Approval

Stage 6 (Continuous Improvement): 
R ).

fff

ff $R$

$\mathrm{R}$

$\mathrm{ff}$

$\mathrm{ff} \mathrm{ff}$

$\mathrm{ff}$

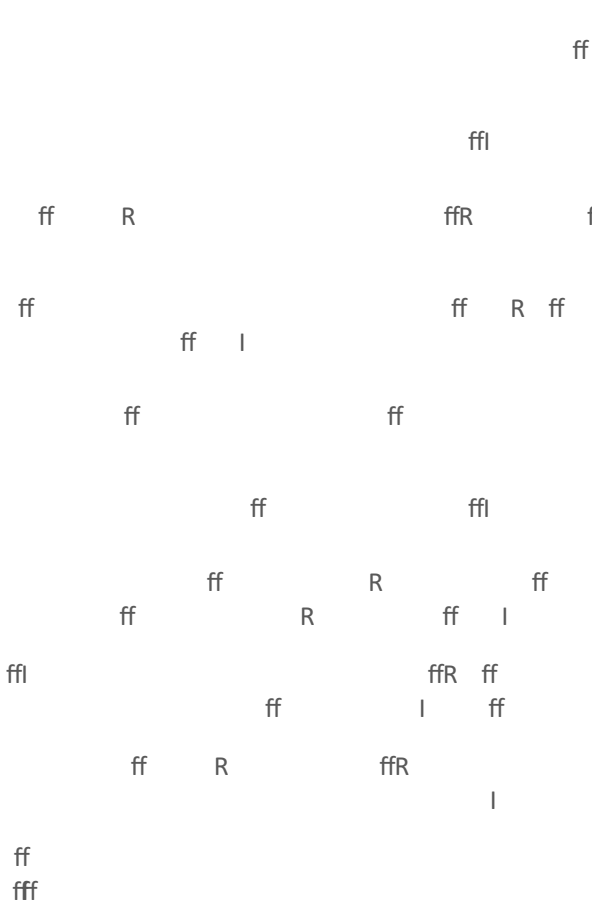

$\mathrm{ff}$

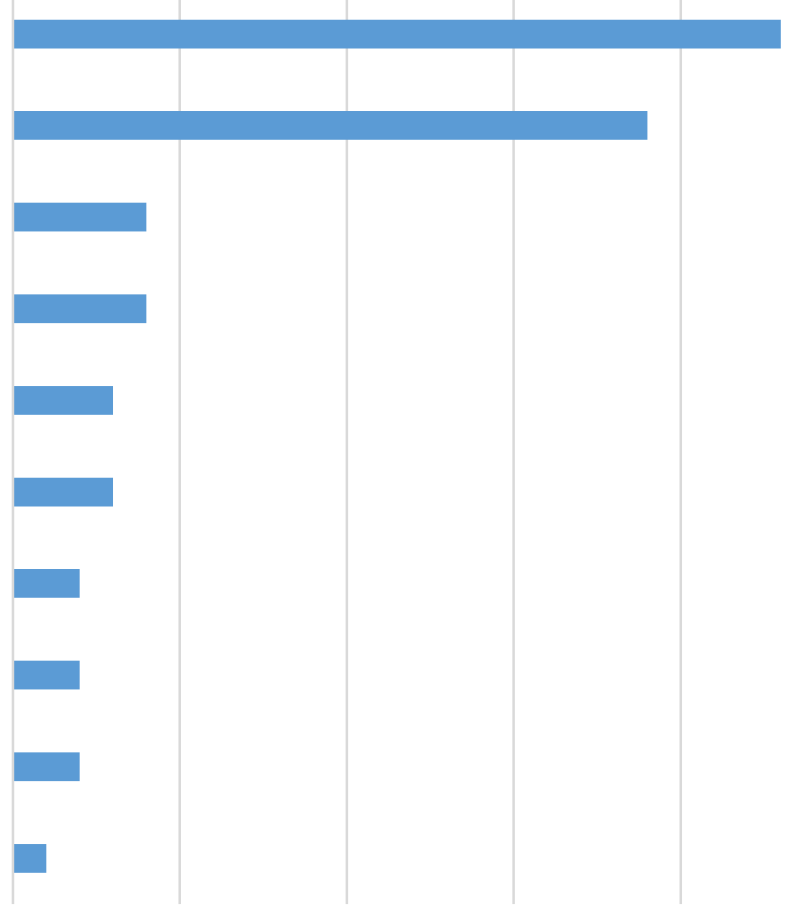

ff

Figure 4. Number of times the external regulations have been referenced in the academic processes

fff

fff

$\mathrm{R}$

ff

ff

- $\mathrm{ff}$

$\mathrm{ff} \mathrm{I}$

- $\quad$ ff

ff

ff $R$

5. Conclusion

R

ff $\quad f f$

ff

fff 
).

$\mathrm{ff}$

fff

ff

ff

References

R ff

ff ff I

fff

ff $R$

ff

ff

ff

ff I

ff

ff

ff

ff

ff

$\mathrm{R}$

I ff

ff 